\title{
Subungual Osteocartilaginous Exostosis
}

THOMAS Y. WOO, M.D. JAMES E. RASMUSSEN, M.D.

Abstract. Subungual exostosis arises underneath the nail plate, originating from the underlying bone. The characteristic appearance of this disorder may occasionally mimic a wide variety of tumors, including subungual verrucae, endochondroma, fibroma, or amelanotic melanoma. With such a wide variety of similar-appearing tumors, optimal treatment of this disorder clearly lies in proper recognition and treatment.

Subungual osteocartilaginous exostosis is a peculiar and distinct type of benign tumor found on the extremities. The location is usually on the toes, especially the hallux, although exostoses arising from the fingers or throughout the entire skeleton have been reported. These disturbances of the underlying bone usually manifest themselves to the dermatologist as flesh-colored or red-brown nodules that project into and disrupt the nail plate. It is the object of this report to bring attention to this interesting condition and to reacquaint clinicians with this rather painful but benign disorder that is readily amenable to surgical excision.

Thomas Y. Woo, M.D., Resident, Department of Dermatology; and James E. Rasmussen, M.D., Professor of Dermatology and Pediatrics, Department of Dermatology and Pediatrics, are from the University of Michigan Medical Center, Ann Arbor, Michigan.

Address reprint requests to James E. Rasmussen, M.D., University of Michigan Medical School, Department of Dermatology, Outpatient Building, Ann Arbor, MI 481090010.

\section{CASE REPORT}

A 9-year-old girl presented to the University of Michigan Department of Dermatology with a slowly enlarging, slightly tender nodule underneath her left first toenail for $2 \frac{1}{2}$ months. She denied trauma to this area. The only symptom was minimal pain and discomfort on ambulation.

On examination, there was an erythematous, redbrown $0.8-\mathrm{cm}$ nodule in the nail bed, near the free edge of the superficial distal medial aspect of her left large toe. There was a minimal amount of fine superficial scaling of the nodule (Fig. 1). Palpation revealed a firm, slightly tender nodule that was fixed to the underlying tissue. The surrounding periungual tissue was erythematous, boggy, and warm without purulent exudate. The rest of the physical examination was normal.

The clinical impression was that of a subungual exostosis. Confirmatory roentgenographic examination of the left hallux revealed an elliptical irregular density, measuring $0.7 \mathrm{~cm}$ at its greatest diameter. It was adjacent to the anterior cortex of the terminal tuft of the first great toe (Fig. 2). The nail was avulsed, an ellipse of overlying skin was removed, and the exostosis was excised followed by curettage of the cortex of the phalanx.

Histopathologic examination of the specimen revealed the histologic features of a subungual exostosis with cancellous bone composed of cellular stroma and trabeculae with calcification. Follow-up of the patient 6 months later revealed partial nail regrowth; the surgical site had healed well, and the toe remained asymptomatic. 


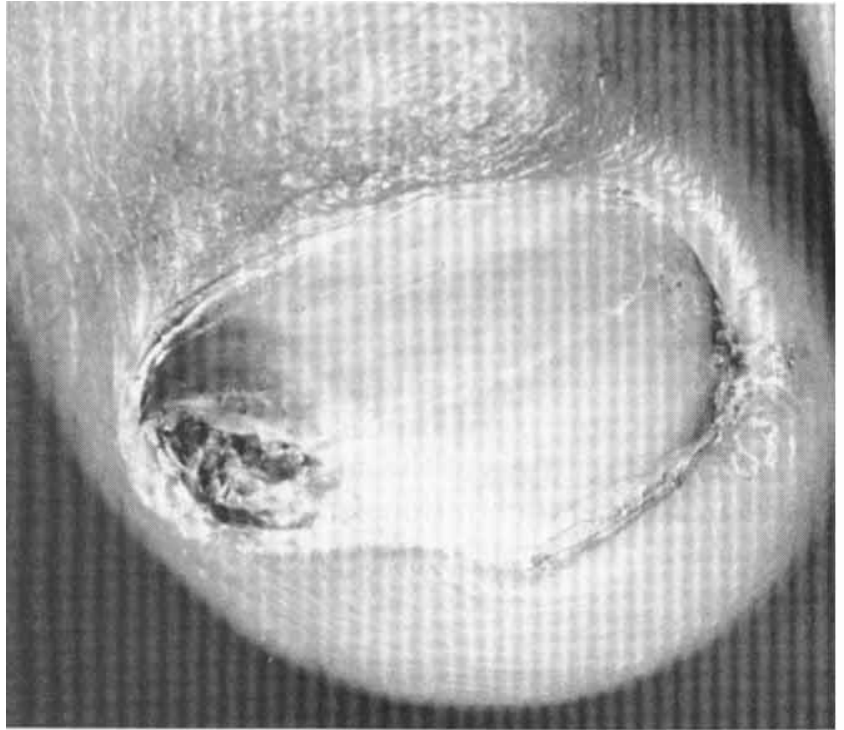

FIGURE 1. A subungual exostosis, appearing as a redbrown nodule that has protruded into the nail plate. $A$ mild paronychia is present.

\section{COMMENTS}

Subungual exostosis was first described by Dupuytren in $1847 .{ }^{1}$ Since then there have been only a handful of reports of this interesting disorder in the dermatologic literature. Exostosis is defined as a bony protruberance projecting outward from the surface of a bone. Subungual exostosis commonly occurs as a solitary lesion occurring on the dorsal aspect of the terminal phalanx of the large toe, usually in adolescents or young adults between the ages of 15 and 30 . It has been reported to involve other toes and occasionally even the fingers ${ }^{2,3}$ and calcaneus. ${ }^{4}$ Females appear to be twice as affected as males. ${ }^{5}$

The etiology of subungual exostosis is unknown. A history of trauma is often obtained but is not necessary for the diagnosis. It has also been suggested that subungual exostosis is a forme fruste of hereditary multiple exostosis or osteochondromas, or even teratologic anomaly arising from the fetal prehallux. The most likely etiology is an inflammatory hyperplasia with metaplasia of cancellous bone arising from the periosteum following irritation, trauma, or infection.

The tumor typically initially appears as a small, firm, pink or brown nodule beneath the free edge of the nail. This may result in mild to considerable pain on walking due to pressure on the nail plate by the expanding tumor. Subsequently, the nail plate becomes pushed upward and may be surgically removed to alleviate any discomfort. The tumor may then expand above the level of the nail bed with an

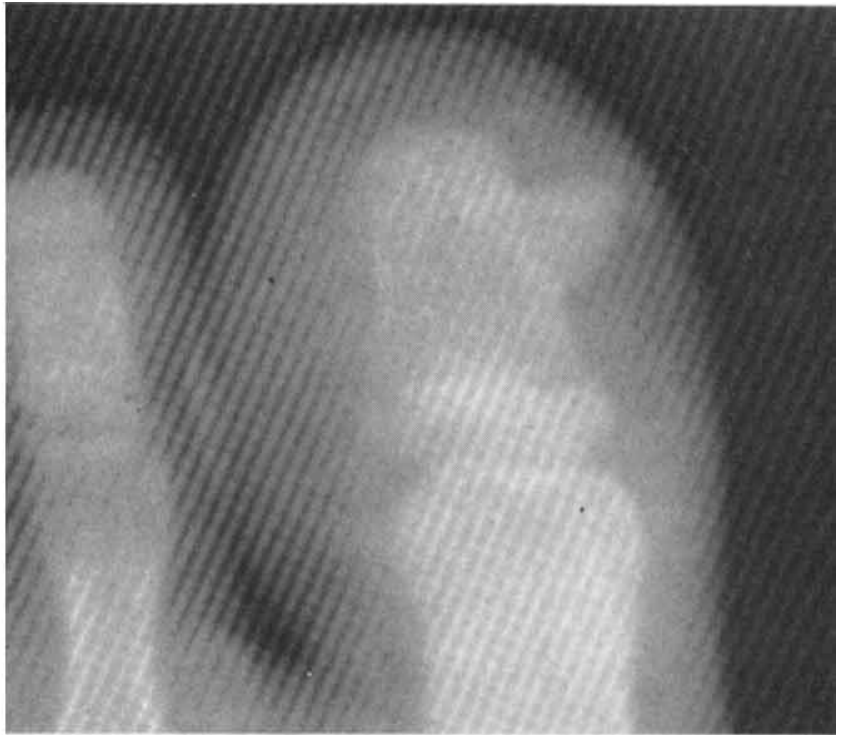

FIGURE 2. Close up of x-ray of left first toe. Note the small projection of bone arising from the terminal tuft.

overlying dense mass of fibrous tissue. It may become verrucous, ulcerated, or eroded. At this point, the differential diagnosis may include subungual wart, pyogenic granuloma, glomus tumor, subungual fibroma, squamous-cell carcinoma of the nail bed, amelanotic melanoma, keratoacanthoma, subungual mucous cyst, and enchondroma.

The latter disorder may especially mimic subungual exostosis due to similar involvement of the distal portion of the phalanx resulting in onychodystrophy and lysis. However, endochondromas are composed of cartilage and arise from the medullary cavity of the bone. As the growth enlarges the cortex becomes thinned and expanded, often resulting in pathologic fractures. An x-ray will differentiate enchondroma as it is characterized by a radiolucent defect associated with a bulbous expansion of the bone. ${ }^{6}$

Radiologic evaluation of a subungual exostosis is diagnostic. One observes a typical small projection of bone, extending outward from the distal tuft of the digit. Occasionally, early lesions, not yet calcified, will yield no radiographic abnormalities. Treatment of a subungual exostosis is total excision. In one series, 43 of 44 patients were treated by local excision with dissection of the lesions and osteotomy at the base. ${ }^{7}$ The fibrocartilaginous cap must be completely removed to prevent recurrence. Local recurrence was seen in $11 \%$ of patients. However, subsequent local re-excision was curative.

In summary, the subungual exostosis is especially important to the dermatologist, as the dis- 


\section{TH ANNUAL OCULOPLASTIC AND FACIAL SURGERY DISSECTION COURSE}

\author{
September 18-22, 1985 \\ New York, New York
}

Sponsored by the International Oculoplastic Society, Inc.

Four intensive days of practical instruction in oculoplastic surgery, including cosmetic blepharoplasty, cosmetic surgery, ptosis and lid reconstruction, lacrimal and facial cancer and facial trauma repair. Special features of the course include videotaped surgical demonstrations and cadaver surgery performed by the registrants. A course syllabus is provided.

Course directors: Pierre Guibor, M.D., Sanford Hecht, M.D., and Eugene Wiggs, M.D.

For more information contact: James F. Pawlak, \% Drs. Guibor, Hecht, and Wiggs, 630 Park Avenue, New York, NY 10021. Telephone: (212)734-1010 or (800)223-4550.

\section{Special Offer!}

A limited number of copies of the issues of the Journal of Dermatologic Surgery and Oncology in Volume 10, 1984 and Volume 9, 1983 are presently available.

If you need to complete your files, simply circle the item(s) below and send your check for the full amount. (Sorry, but we cannot bill for this special offer.)

The usual price for each back issue is $\$ 8.00$, but you may purchase any one of the issues for only $\$ 7.00$. An order for two or more is entitled to an additional $10 \%$ reduction.

Complete the form below, and return it with your check promptly since supplies are limited.

\begin{tabular}{|c|c|c|c|c|}
\hline \multicolumn{2}{|c|}{ Volume 10 (1984) } & \multicolumn{3}{|c|}{ Volume 9 (1983) } \\
\hline No. 1 Jan. & No. 7 July & No. 1 Jan. & No. 7 & July \\
\hline 2 Feb. & 8 Aug. & 2 Feb. & 8 & Aug. \\
\hline March & 9 Sept. & 3 March & 9 & Sept. \\
\hline April & 10 Oct. & $4-$ & 10 & Oct. \\
\hline 5 May & 11 Nov. & 5 May & 11 & Nov. \\
\hline 6 June & 12 Dec. & 6 June & 12 & Dec. \\
\hline
\end{tabular}

I have circled the back issues I need to complete my files and enclose my check for $\$$ The price is $\$ 7.00$ for one issue. I am deducting an additional $10 \%$ from the price since I am ordering ADDRESS

NAME copies. ZIP CODE 